\title{
SULLE OSCILLAZIONI LIBERE DEL LAGO DI CALDONAZZO
}

\author{
P. Caloi - M. C. Spadea
}

1. - Il lago di Caldonazzo si trova all'incirca a $46^{\circ} 1^{\prime}$ Nord, $11^{\circ} 15^{\prime}$ Est. Gr. $\left({ }^{1}\right)$. La sua superficie ha un'altitudine media sul livello del ma-

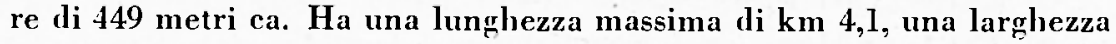
massima di $\mathrm{km} 1$ ed un perimetro di $\mathrm{km} 12$ ca. La sua profondità massima è di m 50 ca.

Il dosso di Tenna lo separa dal Lago di Levico. Gli emissari dei due laghi si uniscono poi per formare il fiume Brenta.

Come per il lago di Levico $\left({ }^{2}\right)$, per la deduzione degli elementi che intervengono nel calcolo, ci siamo valsi di una carta hatimetrica al 5.000 .

II lago fu diviso in quarantun sezioni, con l'equidistanza di $100 \mathrm{~m}$, a partire dall'estremo Sud (Bagni di Caldonazzo). Come al solito, le distanze delle singole sezioni sono state valutate lungo la linea di valle (figg. 1 e 2 ).

La larghezza $b(x)$ in superficie delle singole sezioni, l'area $S(x)$ delle stesse, l'area $v(x)$ della superficie libera del lago fra due sezioni consecutive, l'area $V(x)$ fra le varie sezioni - contate da 1 a 41 e l'estremo Sud del lago e i valori della funzione $\sigma(x)$, pari al prodotto $S(x) \cdot v(x)$, per le 41 sezioni sono riportate nelle tabelle I e III.

La fig. 3 rappresenta la curva normale del lago, la quale, come è noto, dà l'andamento di $\sigma(x)$ in funzione di $V(x)$.

La curva normale essendo risultata piuttosto complessa, si è ritenuto superfluo ricorrere ad uno dei metodi suggeriti da Chrystal, per la determinazione degli elementi idrodinamici del lago.

Ablbiamo senz'altro fatto ricorso al metodo di Hidaka, il quale, come $\dot{c}$ noto, consente di giungere a valori sufficientemente approssimati, qualunque sia la forma della curva normale.

Non staremo naturalmente ad esporre la teoria del metodo, già riassunta ed ampliata in precedenti pubblicazioni $\left({ }^{3}\right)$. Diremo soltanto che il metorlo è stato applicato per il caso $m=2$.

Se indichiamo con a la superficie del lago, $g$ l'accelerazione di gravità e $\lambda$ un parametro che risulta dalla risoluzione di una equa- 


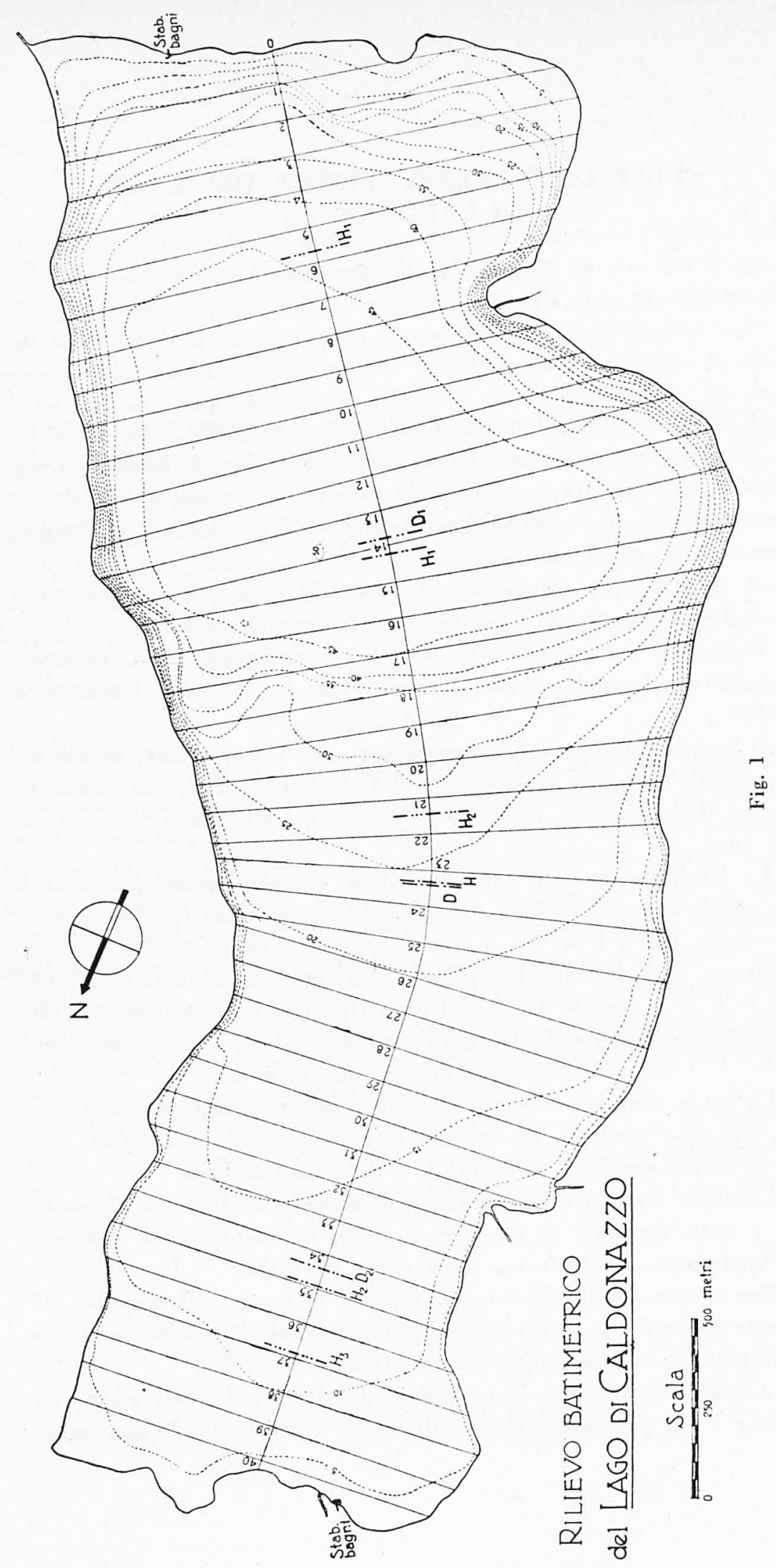


zione di terzo grado. i periodi delle sesse si traggrono dalla formula

$$
T=\frac{2 \pi \cdot a}{\sqrt{g \lambda}} .
$$

L'equazione di terzo grado in $\lambda$, per i vari tipi di oseillazione libera, ha dei coeflicienti ebe risultano da combinazioni di certe gran. dezze $I_{0}, I_{1}, I_{2}, I_{:}, I_{4}$, i cui valori possono essere dedotti dai dati che carattcrizzano la forma del ligo. Questi dati sono contenuti nella tahella I. A causa della complessiti della curva normale, l'equazione di Chrystal è stata risolta mediante integrazione numerica. Gli inteprali risultano avere $i$ seguenti valori:

$$
\begin{aligned}
& I_{v}=0,8873.5 \\
& I_{1}=0,56 \cdot 102 \\
& I_{3}=0,392 \cdot 15 \\
& I_{s}=0,28730 \\
& I_{4}=0,21772 .
\end{aligned}
$$

Con questi valori, l'equazione di terzo grado in $\lambda$ risulta:

$$
58 \lambda^{3}-311 \lambda^{2}+389,7 \lambda-95,24=0 .
$$

Risolta tale equazione con il metodo delle alprossimazioni successive, si sono ottenuti per $\hat{h}$. i scoutenti tre valori:

$$
\begin{aligned}
& \left.\lambda_{1}=0,3223\right] \\
& \lambda_{2}=1,39957 \\
& \lambda_{3}=3,61026 .
\end{aligned}
$$

La superficie del lago vale a $\ldots 5,1744 \mathrm{~km}^{2}$. Conseguono allora dalla [1]

$$
\begin{aligned}
& T_{\mathrm{l}}=9^{\mathrm{m}}, 65 \\
& T_{0}-4^{\mathrm{m}}, 6 \\
& T_{\mathrm{s}}=2^{\mathrm{n}}, 9,
\end{aligned}
$$

che costituiscono i periodi delle sesse uninolale, binodale e trinodale del lagro di Caldonazzo.

1 nodi possono esscre determinati ricorrendo al procedimento indicato in uno dei lavori sopra citati $(3)$, sempre naturalmente nel caso $m=2$.

Per quanto i riferisce all'oscillatzinne libera uninodale, siamo pervenuti alla seguente equazione di tero grato: 


$$
5,172 z^{3}-1,286 z^{2}+0,271 z-1=,
$$

dove $z \grave{e}$ uguale a $\mathrm{V} / \mathrm{a} . z$ quindi è una funzione che può variare tra 0 ed 1. Pertanto, nel caso in esame, una sola delle soluzioni della [2] sarà compresa tra 0 ed 1. Essa vale:

$$
z=0,6394 \text {. }
$$

L'uninodo dista quindi dall'estremo Sud m 2330.

L'equazione dei nodi relativa all'oscillazione libera binodale ̀̀ risultata la seguente:

$$
33,3332 z^{3}-38,6604 z^{2}+7,1070 z+1=0,
$$

le cui soluzioni comprese tra 0 ed 1 sono:

$$
\begin{aligned}
& z_{1}=0,37323 \\
& z_{2}=0,87812 .
\end{aligned}
$$

Se ne deduce che il binodo Sud dista dall'estremo Sud del lago m 1375, mentre il binodo Nord dista dallo stesso estremo m 3490.

Riportiamo qui di seguito l'equazione dei nodi per la sessa tri. nodale:

$$
12,1276 z^{3}-20,1075 z^{2}+9,3412 z-1=0 .
$$

La [4] ha, naturalmente, tutte le radici comprese tra 0 ed 1; esse sono:

$$
\begin{aligned}
& z_{1}=0,1525 \\
& z_{2}=0,5916 \\
& z_{3}=0,9139 .
\end{aligned}
$$

Le distanze dei nodi corrispondenti dall'estremo Sud sono: per il trinodo Sud $m$ 565, per il trinodo medio $m$ 2145, per il trinodo Nord m 3675.

Siamo passati quindi alla determinazione degli spostamenti verticali della superficie del lago, valutati in corrispondenza della linea di valle.

$$
\begin{aligned}
& \xi^{\prime}=-5,172 z^{3}-1,286 z^{2}+0,271 z-1 \text { per la sessa uninodale } \\
& \xi^{\prime \prime}=-33,3332 z^{3}+38,6604 z^{2}-7,1070 z-1 \text { per la sessa binodale } \\
& \xi^{\prime \prime \prime}=-12,1276 z^{3}-20,1075 z^{2}+9,3412 z-1 \text { per la sessa trinodale. }
\end{aligned}
$$

L'andamento degli spostamenti verticali per le tre sesse, di sezione in sezione, prendendo uguale ad 1 lo spostamento iniziale all'estremo 

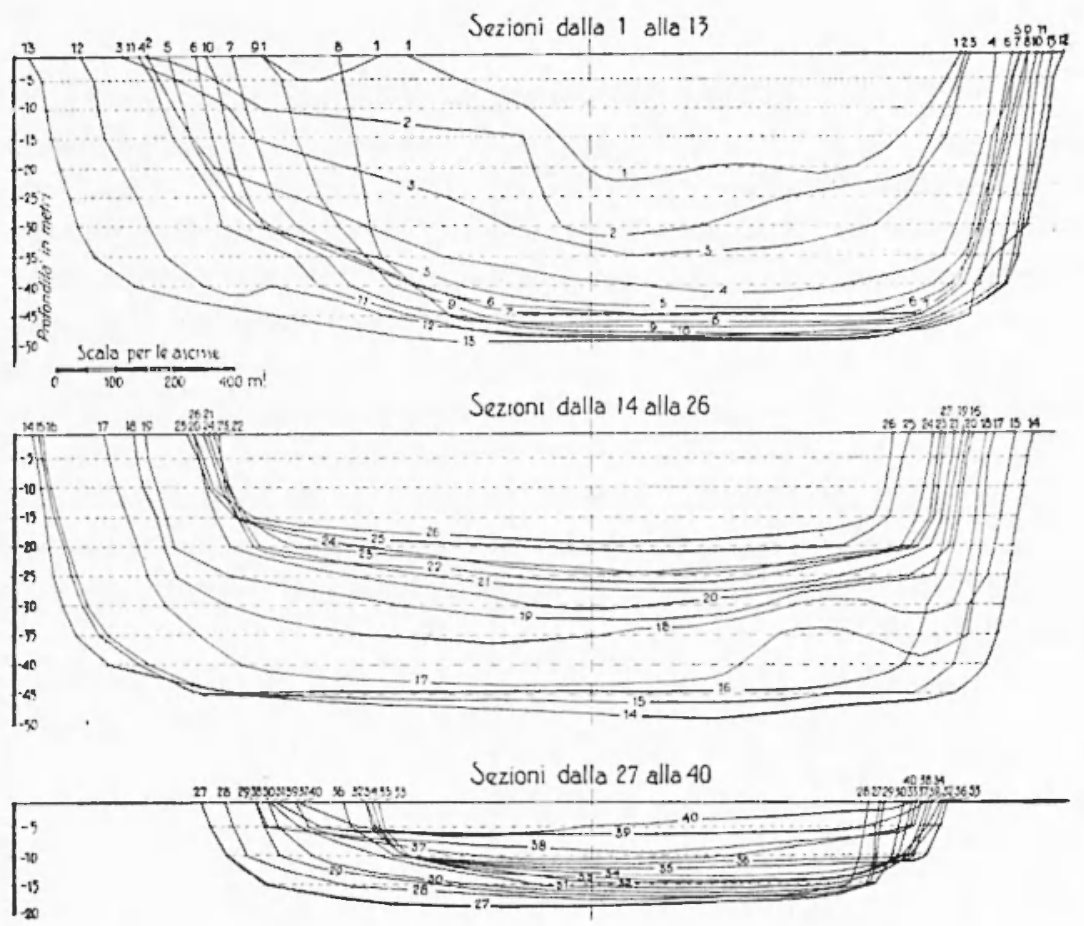

Fig. 2

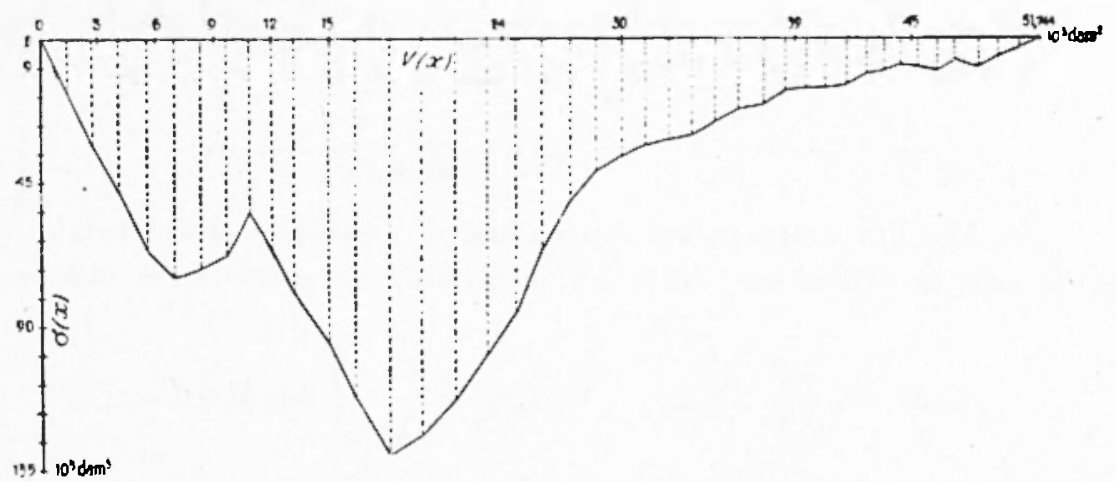

Fig. 3 
Sud, è dato dalla tabella II, ed è graficamente rappresentato nella fig. 4 .

2. - E stato poi applicato il metodo di Defant. Come è noto, tale metodo prende le mosse dalle equazioni differenziali dell'idrodinamica. L'origine delle coordinate viene scelta ad un estremo del lago, la cui superficie lihera costituisce il piano delle coordinate stesse, con l'asse delle $x$ nella direzione dell'asse longitudinale del lago e l'asse delle $y$ ad essa normale. L'asse delle $z$ è diretta positivamente verso l'alto. Se $x$ rappresenta l'ascissa di un punto $P$ del lago, $S(x)$ la sezione trasversale, $b(x)$ la larghezza del lago nel punto considerato, la massa d'acqua tra la sezione Sixi e la sezione successiva $\tilde{S}(\boldsymbol{x}+\boldsymbol{d} \boldsymbol{x})$ sari espressa da $S(x) d x$. Se gli strati di spessore $d x$ compiono uno spostamento orizzontale pari a $\xi$ ed uno verticale uguale ad $\eta$, dalle equazioni di moto e di continuità dell'idrodinamica, si traggono le equazioni differenziali

$$
\frac{\partial^{?} \xi}{\partial t^{i}}=\underline{\underline{y}} \frac{\partial}{\partial x}\left[\frac{1}{b(x)} \cdot \frac{\partial S(x) \cdot \xi}{\partial x}\right], \eta=-\frac{1}{b(x)} \cdot \frac{\partial}{\partial x}(S(x) \cdot \xi) .
$$

La prima può essere messa sotto la forma

$$
\frac{\partial^{2} \xi}{\partial t^{0}}=\cong \frac{\partial \eta}{\partial x} \text {. }
$$

Le soluzioni di questa equazione sono funzioni periodiche di $t$ e si possono scrivere

$$
\xi=\xi_{0}(x) \cos \left(\frac{2 \pi}{T} t+\varepsilon\right), \eta=\eta_{0}(x) \cos \left(\frac{2 \pi}{T} t+\varepsilon\right) .
$$

Le ampiezze dei moti orizzontali e verticali, dipendenti solo da $x$ e non da $t$ e che noi indicheremo con $\xi_{0}, \eta_{0}$, soddisfano allora alle relazioni

$$
\frac{4 \pi^{2}}{T^{2}} \xi_{0}=g \frac{d \eta_{0}}{d x} \quad, \quad \eta_{0}=-\frac{1}{b(x)} \frac{d}{d x}\left(S(x) \cdot \xi_{0}\right) .
$$

Sostituendo nella prima equazione al quoziente differenziale il quoziente di differenze finite ed integrando la seconda, si ottiene:

$$
2 \Delta \eta_{0}=\frac{4 \pi^{2}}{g T^{2}} 2 \xi_{0} \Delta x \quad, \quad 2 \xi_{0}=-\frac{1}{S(x)} \int_{0}^{x} 2 \eta_{0} b(x) d x .
$$

Nella $[5], 2 \eta_{0}$ e $2 \xi_{0}$ sono le ampiezze delle oscillazioni lungo il lago, 


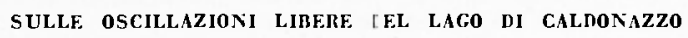

19

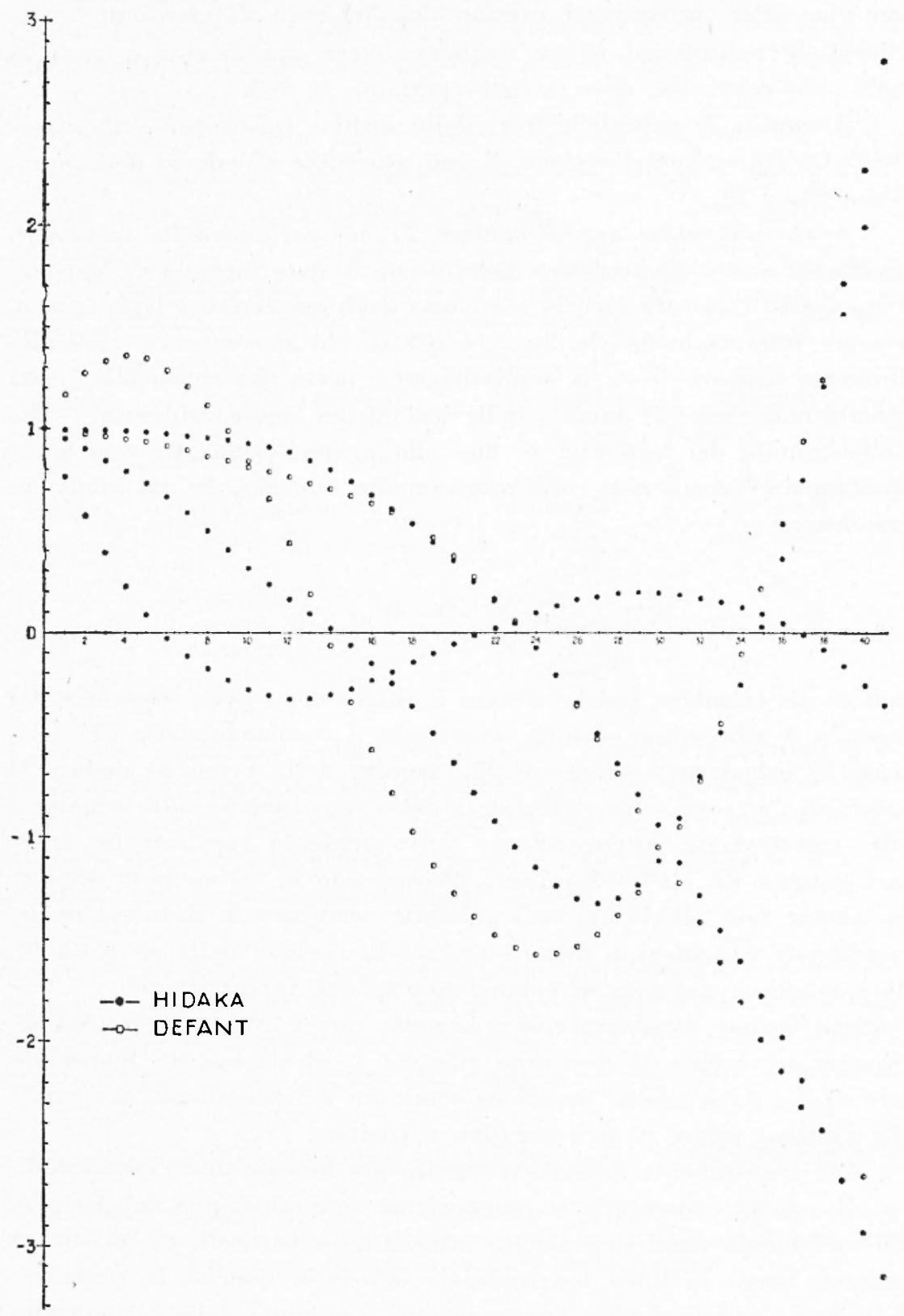

Fig. 4 
nei due sensi verticale ed orizzontale. Nel caso di masse di acqua chiuse, le oscillazioni libere debbono avere spostamenti orizzontali nulli alle estremita, dove avremo pertanto $\xi=0$.

Assegnate le superficie $S(x)$ delle sezioni trasversali e le superficie $v(x)$ fra sezione e sezione, si può procedere al calcolo delle grandezze $2 \eta_{0}$ e $2 \xi_{0}$.

Avuto un valore approssimativo $T_{1}$ del periodo della sessa uninodale (che può anche essere dedotto con la nota formula di Merian), resta fissato il fattore $4 \pi^{2} / T_{1}=$. Ad uno degli estremi del lago $(x=0$, sezione trasversale 0 , sia $2_{\|}=+100 \mathrm{~cm}$ lo spostamento verticale. Dovremo supporre - e la supposizione è tanto più vicina alla veritì quanto maggiore è il numero delle sezioni del lago considerato — che dall'estremità del lago $(x=0)$ fino alla prima sezione trasversale, lo spostamento considerato resti praticamente costante. In tal morlo, la grandezza

$$
q=\int_{0}^{\mathrm{x} l} 2 \eta_{0} \cdot b(x) d x=2 \eta_{0} \cdot v\left(x_{1}\right)
$$

può essere calcolata, poiché ì noto il valore $v\left(x_{1}\right)$ della superficie del lago da 0 alla prima sezione trasversale. La seconda delle [5] consente di calcolare il valore di $2 \xi_{0}$, mentre dalla prima si deduce il valore di $2 \eta_{0}$ variazione dell'altezza dello spostanento dalla sezione 0 alla sezione 1 , in corrispondenza della quale lo spostamento stesso sarà espresso da $\left(100+2 \psi_{0}\right) \mathrm{cm}$. Procedendo in tal modo di sezione in sezione fino all'ultima, sara possibile verificare se il valore di $T_{1}$ considerato concideri o meno con l'esatto periodo della sessa uninodale del lago: nel caso di coincidenza infatti, in corrispondenza dell'ultima sezione, considerata di area nulla, dovrà aversi $q=0$. Quando ciò non si verifica, si dovranno ripetere $i$ calcoli con un nuovo valore di $T_{1}$, da scegliersi maggiore o minore del precedente a seconda che l'ultimo valore di $q$ è negativo o positivo.

Un procedimento analogo si seguirà per le sesse di 2, 3, ecc. nodi.

11 metodo consente la contemporanea determinazione del periodo, dell'andamento degli spostamenti orizzontali e verticali, di sezione in sezione, lungo la linea longitudinale del lago, nonché la posizione delle linee nodali. Anche questo metodo prescinde dalla forma della curva normale.

Abbiamo applicato il metodo di Defant per la determinazione degli elementi relativi alle oscillazioni libere uninorlali e hinodali. 
Per quanto concerne la sessa uninodale, con un periodo $T=9^{\mathrm{m}}, 7$, si is avuto un residuo $q=2,81.10^{10} \mathrm{~cm}^{3}$. Possiamo concluderne che il periodo della sessa uninodale, calcolato con il metodo di Defant ì dell'ordine di $9^{\mathrm{m}}, 65$.

La tabella III riporta pure i risultati relativi alla sessa binodale.

Nel prospetto che segue sono messi a confronto i risultati ottenuti con i due metodi di Hidaka e Defant:

\begin{tabular}{|c|c|c|c|}
\hline \multicolumn{2}{|c|}{ Oscillazioni libere } & $\begin{array}{c}\text { Primo Metodo } \\
\text { (Hidakil) }\end{array}$ & $\begin{array}{c}\text { Secondo Metodo } \\
\text { (Defint) }\end{array}$ \\
\hline uninodale & $\left\{\begin{array}{c}\text { periodo } \\
\text { nodo (dall'estremo } \\
\text { Sud) }\end{array}\right.$ & $\begin{array}{c}9^{m}, 65 \\
2330 \text { metri }\end{array}$ & $\begin{array}{c}9 m, 65 \\
2340^{m} \text { metri }\end{array}$ \\
\hline binodale & $\begin{array}{ll}\text { periodo } \\
\text { I binodo } \\
\text { II linodo }\end{array}$ & $\begin{array}{l}4^{\mathrm{m}}, 6 \\
1375 \text { metri } \\
3490 \text { metri }\end{array}$ & $\begin{array}{l}4^{\mathrm{m}}, 8 \\
] 120 \text { metri } \\
3440 \text { metri }\end{array}$ \\
\hline trinodale & $\left\{\begin{array}{l}\text { periodo } \\
\text { trinodo Sud } \\
\text { trinodo medio } \\
\text { trinodo Nord }\end{array}\right.$ & $\begin{array}{l}2^{m .9} \\
565 \text { metri } \\
21.45 \text { metri } \\
3675 \text { metri }\end{array}$ & \\
\hline
\end{tabular}

Si nota subito che $i$ due metodi hanno condotto a risultati praticamente coincidenti, ciò che si verifica piuttosto di rado. Questo è in parte dovuto al fatto che la forma del lago non si discosta molto da quella prevista dalla teoria ed in parte dall'aver diviso il lago in un numero di sezioni sufficientemente rrrande.

3. - Ancora non sono state compiute osservazioni sistematiche sui moti liberi del lago di Caldonazzo. Solo il prof. Polli ebhe a fare qualche saltuaria registrazione di sesse del lago in questione. Quella riportata nella fig. 5 è appunto un esempio di registrazione, ottenuto

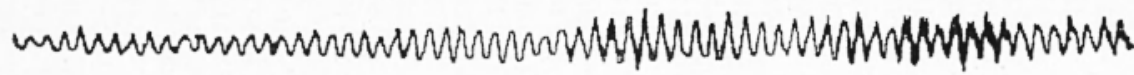

Pig. 5 - Sesse uninodali del lago di Caldonazzo registrate con limnografo Polli dalle $19430 m$ ca. del 10 aprile 1950 alle $7^{\text {h }}$ ca. dell'll aprile 1950 , presso i Bagni di Caldonazzo. (Ridotta a (a. i 35 delloriginale).

facendo stazione presso i Bagni di Caldonazzo, con un apparecchio ideato dallo stesso prof. Polli.

Come media di oltre $17^{1 /}$ di registrazione, il periodo osservato per 


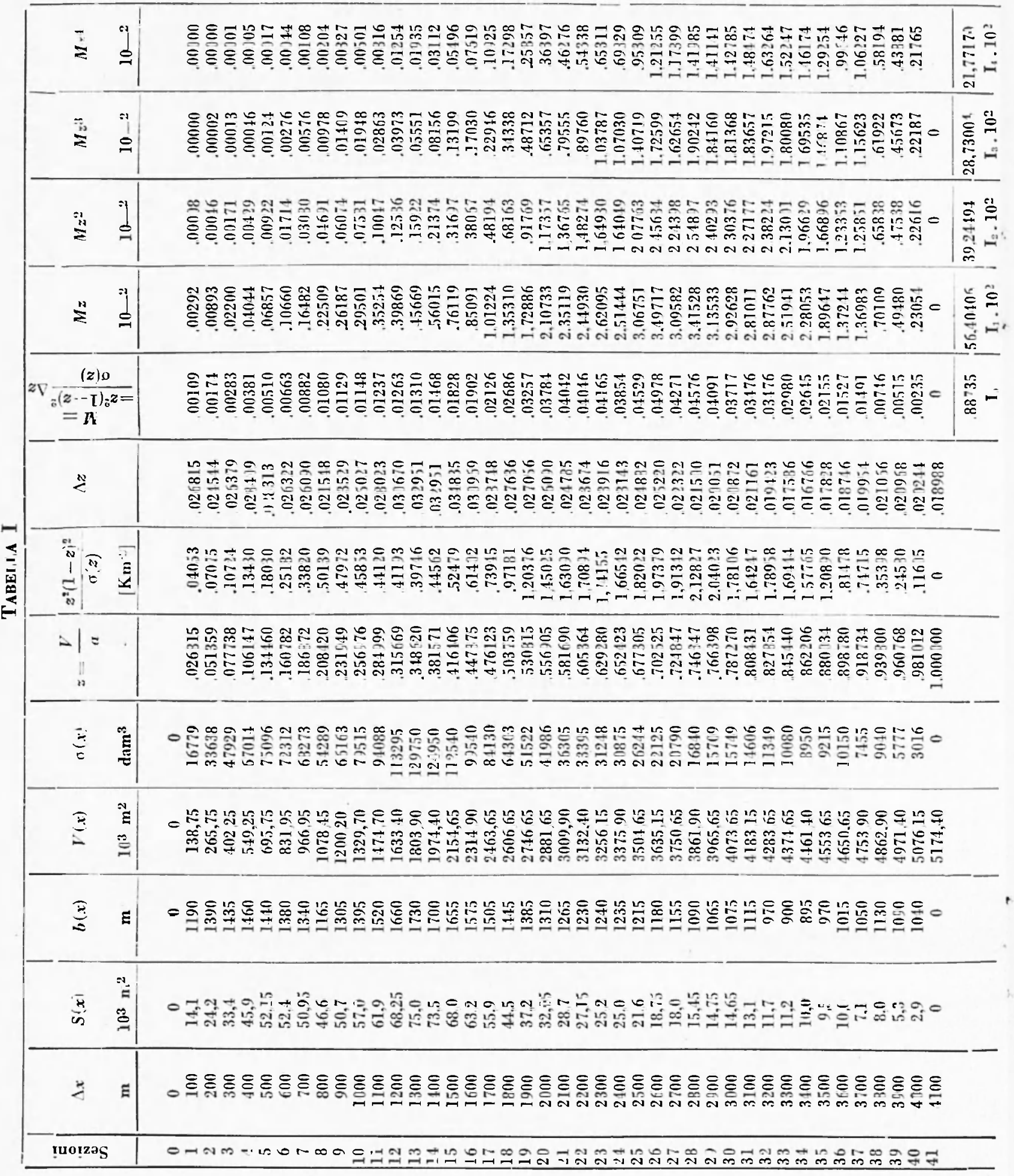




\section{Tabella II}

\begin{tabular}{|c|c|c|c|}
\hline Sezioni & $\zeta^{\prime}$ & $\varphi^{\prime \prime}$ & $\zeta^{\prime \prime \prime}$ \\
\hline 0 & -1.0 & -1 & $1-$ \\
\hline 1 & -.994 & $-1,163$ & -.764 \\
\hline 2 & -.989 & $-1,268$ & -.572 \\
\hline 3 & -.984 & $-1,334$ & -.390 \\
\hline 4 & -.979 & $-1,359$ & -.220 \\
\hline 5 & -.974 & $-1,338$ & -.078 \\
\hline 6 & -.968 & $-1,282$ & +.033 \\
\hline$i$ & -.960 & $-1,196$ & +.122 \\
\hline 8 & -.953 & $-1,108$ & +.183 \\
\hline 9 & -.942 & -.984 & +.236 \\
\hline 10 & -.927 & -.839 & +.278 \\
\hline 11 & -.907 & -.657 & +.310 \\
\hline 12 & -.880 & -.440 & +.327 \\
\hline 13 & -.843 & -.191 & +.326 \\
\hline 14 & -.769 & +.065 & +.310 \\
\hline 15 & -.737 & $\begin{array}{r}+.338 \\
+\end{array}$ & +.279 \\
\hline 16 & -.673 & +.574 & +.240 \\
\hline 17 & -.60 .4 & +.782 & +.198 \\
\hline 18 & -.529 & $\begin{array}{r}+.969 \\
\end{array}$ & +.153 \\
\hline 19 & -.445 & +1.135 & +.107 \\
\hline 20 & --.355 & +1.275 & +.061 \\
\hline 21 & -.259 & +1.386 & +.017 \\
\hline 22 &.- .160 & +1.471 & -.023 \\
\hline 23 & -.050 & +1.530 & -.062 \\
\hline 24 & +.066 & +1.562 & -.096 \\
\hline 25 & +.201 & +1.564 & -.129 \\
\hline 26 & +.349 & +1.530 & -.156 \\
\hline 27 & +.490 & +1.466 & -.175 \\
\hline 28 & +.636 & +1.373 & -.187 \\
\hline 29 & +.781 & +1.256 & -.192 \\
\hline 30 & +.910 & +1.102 & -.191 \\
\hline 31 & +1.111 & +.909 & -.182 \\
\hline 32 & +1.277 & +.700 & -.167 \\
\hline 33 & +1.435 & +.482 & -.146 \\
\hline $3 . t$ & +1.593 & +.247 & -.120 \\
\hline 35 & +1.767 & -.032 & -.086 \\
\hline 36 & +1.960 & -.359 & -.042 \\
\hline 37 & +2.174 & -.746 & +.015 \\
\hline 38 & +2.412 & -1.202 & +.086 \\
\hline 39 & +2.660 & -1.704 & +.169 \\
\hline 40 & +2.911 & -2.236 & +.262 \\
\hline 41 & +3.157 & -2.780 & +.361 \\
\hline
\end{tabular}




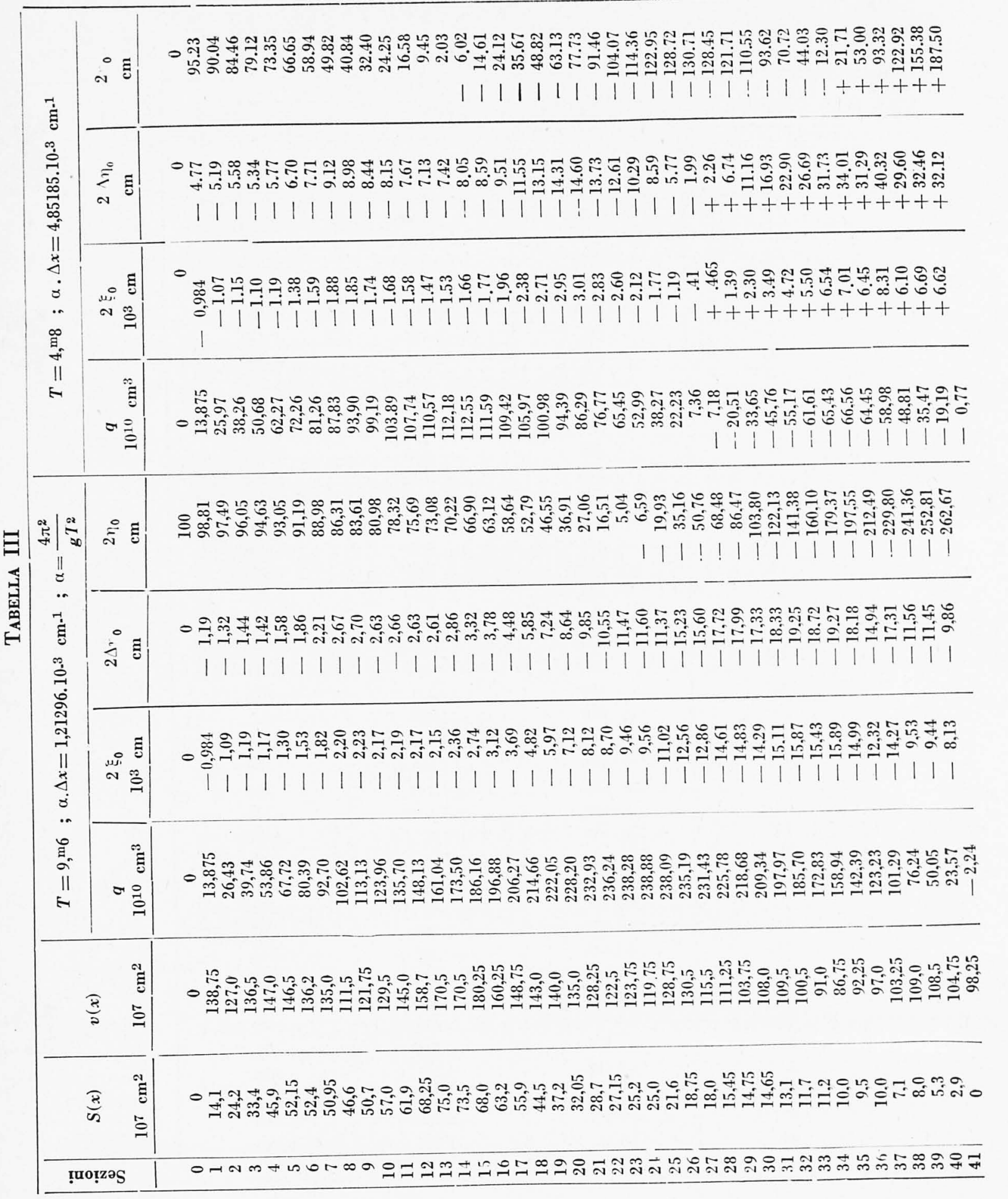


la sessa uninodale è risultato di ca. $10^{\mathrm{m}}$ : gli esempi più netti però e y'iù numerosi, hanno periodi di $9^{\mathrm{m}}, 5$, che risulta pertanto il valore predominante.

L'accordo con i valori calcolati non potrebbe essere migliore. Ad ogni modo, le osservazioni sistematiche che ci proponiamo di fare ci consentiranno di precisare ulteriormente il valore del periodo osservato.

$$
\text { Roma - Istituto Nazionale di Geofisica - Agosto } 1951 .
$$

\section{RIASSUNTO}

É stato fatto, per il lago di Caldonazzo, un lavoro analogo a quello compiuto per la ricerca delle oscillazioni libere del lago di Levico.

In questo primo contributo vengono riportati $i$ risultati dell'applicazione di due diversi metodi analitici per la determinazione dei periodi, dei nodi e dell'andamento delle ampiezze relativi alle prime tre sesse del lago.

I risultati sono in ottimo accordo tra loro; accordo che molto raramente si realizza.

Per quanto riguarda la sessa uninodale, viene riportato un esempio di registrazione, che dà, per i periodi, valori conformi a quelli ottenuti con il calcolo.

$\grave{E}$ nostra intenzione completare il lavoro con una sistematica serie di osservazioni, che ci riserviamo di eseguire non appena possibile.

\section{SUMMARY}

The same research on free oscillations of the water, which had been effected for the Levico Latie, has been effected also for the Caldonazoo Lake.

In this first article are reported the results obtained by the application of tuo different analytical methods for the period determination, as well as for the determination of nodes and amplitude variations referred to the three first seiches of the lake.

Results match very satisfactorily, though such a concordance is rather exceptional.

As for the uninodal seiche, a recording example has been reported, 
which gives recorded period values agreeing with the values obrained by calculation.

We infend to complete our exposition by reporting a systematic series of observations which we will publish as soon as possible.

\section{BIBLIOGRAEIA}

(1) Riccardi R.: I laghi d'Thalia. Boll. della Soc. Geog. Ital., Roma 1925.

(2) Calor P.: Oscillazioni libere del lago di Letico. Annali di Geof. IV, 2, 1951.

(3) Calor P.: Le sesse del Lago di Gardr. Parte I e II, Annali di Geof. I, 1 e 2, 1948. 